\title{
Author's reply to letter from Matthes and Zucca-Matthes on "Quantitative analyses of variability in normal vaginal shape and dimension on MR images"
}

\author{
Jiajia Luo $^{1,2}$ • Cornelia Betschart ${ }^{1,3}$ • James A. Ashton-Miller ${ }^{1,2}$ • John O. L. DeLancey ${ }^{1,4}$
}

Published online: 15 August 2016

(C) The International Urogynecological Association 2016

\section{Dear Editor}

We appreciate this interesting commentary by Matthes et al. The issue raised in this letter [1] concerns differences between vaginal length and penile length. In considering this issue, it is important to point out that the measurement techniques and the landmarks used differ. In the review of penile measurements by Veale et al. [2], length is measured from "the root (pubopenile junction) of the penis to the tip of the glans ... where the pre-pubic fat pad was pushed to the bone." Our measurements are made from the level of the hymen, which is several centimeters above the vulva [3]. In addition, depending on sexual position, the pubic bones may limit how far above the hymen the penis reaches. What is unknown is the distance from the penile root to the hymen, and so an offset of some magnitude must be taken into account in assessing these dimensions.

A second factor that the writers point out is the distensibility of the vagina. The vagina is one of the most stretchable organs in the body, as evidenced by its ability to accommodate a full-term infant. Considering vaginal length at rest in the

This reply refers to the comment available at doi: 10.1007/s00192-0163094-5.

John O. L. DeLancey

delancey@umich.edu

1 Pelvic Floor Research Group, University of Michigan, Ann Arbor, MI 48109, USA

2 Department of Mechanical Engineering, University of Michigan, Ann Arbor, MI 48109, USA

3 Department of Gynecology, University Hospital of Zurich, 8091 Zurich, Switzerland

4 Division of Gynecology, Department of Obstetrics and Gynecology, University of Michigan, Ann Arbor, MI 48109, USA undistended state is only part of a full picture, and the degree to which it can be distended both short term and long term, as the commenters point out, is a factor as well and should not be considered only at a single time point. Although at first there may be some disproportion between the female and male sexual organs, the vagina can become longer with repeated intercourse. This is most dramatically illustrated in some young women with Müllerian agenesis who have a vaginal length of only 1 or $2 \mathrm{~cm}$ who are found after repeated attempts at vaginal penetration to have developed a functional vagina of 7 or $8 \mathrm{~cm}$ in length.

As the letter points, out there are instances when the penis is too long for the vaginal length in the short term. In extreme cases, vaginal rupture from too-deep penetration during intercourse can occur, but these instances are so uncommon that case reports are still written about this situation. Certainly, short of this dramatic consequence of size mismatch, a "relative short vagina syndrome" certainly can exist and can be a factor in painful intercourse. However, because of the factors listed above, care must be taken in using measurements that have different landmarks as a zero point in order to make this diagnosis.

\section{References}

1. Matthes Â, Zucca-Matthes G. A comment about the quantitative analysis of normal vaginal shape and dimension. Int Urogynecol J.

2. Veale D, Miles S, Bramley S, Muir G, Hodsoll J. Am I normal? A systematic review and construction of nomograms for flaccid and erect penis length and circumference in up to $15,521 \mathrm{men}$. BJU Int.

2015;115(6):978-86

3. Luo J, Betschart C, Ashton-Miller JA, DeLancey JO. Quantitative analyses of variability in normal vaginal shape and dimension on MR images. Int Urogynecol J. 2016. doi:10.1007/s00192-016-2949-0. 